\title{
ARGAMASSA ADERIDA, 0 ELO FRACO NO CONCRETO RECICLADO: UMA REVISÃO
}

ADHERED MORTAR, THE WEAK LINK IN RECYCLED CONCRETE: A REVIEW

\author{
DANILLO DE ALMEIDA E SILVA, MSC. | UnB \\ CARLOS EDUARDO LUNA DE MELO, Dr.| UnB
}

\begin{abstract}
RESUMO
Um grande avanço tecnológico tem sido alcançado no uso do agregado reciclado para produção de novos concretos. Para tanto, pesquisas têm sido realizadas com o objetivo de dar uma destinação mais sustentável aos resíduos da construção, mitigando a problemática do uso deste material para produção de concretos estruturais, não estruturais e materiais construtivos. Estudos relatam que a argamassa aderida (AD) ao agregado reciclado é o elo fraco na microestrutura deste material. 0 objetivo da pesquisa é relatar o avanço tecnológico realizado com o agregado reciclado para concreto e seus fatores influentes, tais como granulometria, forma, textura, densidade e argamassa aderida ( $A D)$, além de demonstrar que esses fatores podem ser controlados. Dentre os mecanismos utilizados para melhorar as propriedades físicas e mecânicas do agregado reciclado, estão os tratamentos térmicos, químicos e mecânicos. Estas pesquisas demostram que o agregado reciclado pode vir a ser utilizado em concretos estruturais, dando enfoque na produção desse material tão promissor com um custo baixo e com elevado ganho ambiental.
\end{abstract}

PALAVRAS-CHAVE: Agregado reciclado; Argamassa aderida; Concreto reciclado, Sustentabilidade.

\begin{abstract}
A great technological advance has been achieved in the use of recycled aggregate to produce new concretes. Therefore, researches have been carried out with the objective of giving a more sustainable destination to construction waste, mitigating the problem of using this material for the production of structural concrete, non-structural and constructive materials. Studies report that the mortar adhered $(A D)$ to the recycled aggregate is the weak link in the microstructure of this material. The objective of the research is to report the technological advances made with recycled aggregate for concrete and its influencing factors, such as particle size, shape, texture, density and bonded mortar (AD), in addition to demonstrating that these factors can be controlled. Among the mechanisms used to improve the physical and mechanical properties of recycled aggregate are thermal, chemical and mechanical treatments. These researches demonstrate that recycled aggregate can be used in structural concrete, focusing on the production of this very promising material with a low cost and high environmental gains.
\end{abstract}

KEY WORDS: Recycled aggregate; Adhered mortar; Recycled concrete, Sustainability. 


\section{INTRODUÇÃO}

0 concreto é o produto mais consumido no mundo (MEHTA; MONTEIRO, 2008), depois da água e da energia elétrica. É constituído por cimento, agregados, água e, eventualmente, aditivos. Cerca de $70 \%$ a $85 \%$ do concreto é composto pelos agregados graúdo e miúdo, o que os tornam os insumos mais produzidos $\mathrm{e}$ consumidos em todo o mundo (ALMEIDA, 2016), (SILVA, 2012), sendo, consequentemente, uns dos produtos mais poluentes desde a sua extração, beneficiamento e seu descarte na forma de resíduo de construção e demolição RCD.

As atividades de construção demandam uma notável quantidade de recursos naturais, tais como argila, areia e cascalho, que usualmente são fornecidos por meio da extração de jazidas próximas aos centros urbanos (BRASILEIRO; MATOS, 2015). Além do crescente consumo destes recursos, a grande produção de RCD tem provocado mudanças na sociedade e nas legislações.

As diretrizes, como a resolução $\mathrm{n}^{\circ} 307$ do CONAMA (2002) e da Política Nacional de Resíduos Sólidos (PNRS, 2010), têm levado à consolidação de técnicas de reciclagem como alternativa viável para a sustentabilidade da construção e do meio ambiente.

Segundo estudos realizados por Silva e Melo, (2020b) somente menos de $10 \%$ do resíduo gerado na construção e demolição tem um tratamento adequado. Este ocorre por meio da coleta, separação, processamento e destinação para confecção de novos produtos, como blocos estruturais, blocos para vedação, blocos para piso, agregado graúdo e agregado miúdo artificial para concreto e argamassa.

Diversos autores vêm desenvolvendo estudos com RCD e agregados reciclados de concreto ARC, na tentativa de minimizar os efeitos negativos decorrentes das propriedades físico mecânicas deste material, utilizando-se para isso tratamentos dos mais variados.

De acordo com Santana et al. (2011) o concreto reciclado já vem demonstrando uma boa atuação numa série de usos em obras urbanas, com a obtenção de custos bastante benéficos. É possivel programar sua utilização em concretos para bases de pavimentos e produção de artefatos pré-moldados em concreto (tubos, lajes, blocos).

A necessidade de desenvolver concretos com agregados não convencionais é urgente por razões ambientais e econômicas. Pesquisas realizadas mostram que os resíduos industriais e outros subprodutos foram adicionados na fabricação de cimentos "aglomerantes hidráulicos" utilizados para fabricação de concretos com o objetivo de melhorar as propriedades destes e reduzir seu custo (BASTOS; CRUZ; WOELFFEL, 2016).

Atualmente, a proposta de incorporar o resíduo de construção e demolição reciclado RCD-R como agregado no concreto e materiais de construção não é uma alternativa, e sim um objetivo para a sustentabilidade da construção civil (SILVA; MELO, 2021), (BASTOS; CRUZ; WOELFFEL, 2016), (KATZ, 2004), (CONAMA, 2002).

Um dos problemas relacionados ao uso do RCD-R na construção civil está ligado à sua coleta $e$ separação na fonte geradora. Uma política cultural está sendo introduzida nos canteiros de obras, com a finalidade de conscientização dos profissionais, com o objetivo de minimizar o descarte inadequado destes materiais (BASTOS; CRUZ; WOELFFEL, 2016).

\subsection{Materiais que original o resíduo de construção}

As origens dos RCD advêm de diferentes etapas e componentes da construção civil, como fundação, estrutura de concreto, argamassas, elementos de vedação e revestimentos, podendo ser gerado em todas as fases de uma obra. A reciclagem de entulho de obras de construções tem o objetivo de preservar o meio ambiente, suprindo a demanda de novos materiais e dando destinação sustentável aos resíduos, que seriam descartados em aterros ou despejados de forma irregular na margem de rios e córregos.

Os entulhos de concretos e argamassa são classificados como classe A (CONAMA, 2002), e são passíveis de reciclagem, podendo ser classificados como agregados graúdos e miúdos: "areia e brita". E dentre os materiais do RCD, os concretos são os mais promissores para reciclagem e produção de agregados para a Indústria da Construção Civil.

\subsection{Resíduos de construção como agregado para construção civil}

A Diante da escassez dos recursos naturais e de custos cada vez mais elevados de insumos utilizados na construção civil, somados aos altos índices de desperdício da construção civil, gerando resíduos que na maioria das vezes são descartados de forma inadequada, poluindo 
nascentes, rios e lagos, torna-se necessária uma adequada destinação dos RCD.

Estudos realizados por Almeida e Luz (2009) revelam que a brita (agregado graúdo), representa em média $2 \%$ do custo global de uma edificação e $60 \%$ do seu volume. A demanda por agregados da construção civil no Brasil partiu de 460 milhões de toneladas em 1997 para 673 milhões de toneladas em 2011, crescimento correspondente a $46,2 \%$, e um crescimento anual de $2,8 \%$ a.a. Outro dado mais recente é relatado em pesquisa realizada pela ANEPAC - Associação Nacional das Entidades de Produção de Agregados para Construção Civil, na qual estimase um consumo de 514 milhões de toneladas em 2019 (METSO, 2019).

Inúmeros estudos examinaram a possibilidade do uso do agregado reciclado de concreto e cerâmica reciclada advindos da construção e demolição como fontes de matéria prima para uso em novos materiais (SILVA; DE BRITO; DHIR, 2014), (AMORIM; BRITO, 2012), (GOMES; DE BRITO, 2007). Outras pesquisas abordam também o uso do agregado reciclado como agregado graúdo e agregado miúdo para uso em concretos (STROHER et al., 2017), (ALVES et al., 2014;).

Pesquisadores como Fernandes e Melo (2020), Martins, Correia e Brito (2016), Bravo et al., (2015), Amorim e Brito (2012), Gomes e Brito (2007) apresentam resultados promissores com o uso do agregado reciclado de concreto para uso em novos produtos de concreto pré moldado como guias, blocos e pisos permeáveis.

Novos produtos e componentes construtivos utilizando o agregado reciclado é um caminho promissor para a sustentabilidade da construção civil. Por meio da reciclagem pode se obter material e componente adequado à utilização em diversos serviços de construção, além do fator economia, pois em geral fica mais barato reciclar os resíduos de $R C D$, do que gerenciar sua disposição final em aterros ou a sua remoção de locais irregulares (KLEIN; DIAS, 2017), (SACHO et al., 2016), (TROCA, 2007).

\section{A PROBLEMÁTICA DO USO DO RESÍDUO DE CONSTRUÇÃO COMO AGREGADO}

0 presente artigo busca referenciar a problemática do uso do agregado reciclado para concreto e os mecanismos relacionados à sua produção e qualidade, fatores que são preponderantes em seu desempenho.

Dentre esses fatores está a determinação da composição do RCD e o conhecimento da composição da amostra deste RCD utilizado, que é de primordial importância para a qualidade do concreto com ele produzido.

0 agregado reciclado misto ARM é mais acessível e produzido no Brasil, devido à falta de coleta seletiva ou falta de planejamento nos empreendimentos de demolição e construção. Atualmente, a coleta é feita por caçamba nos canteiros de obra espalhados nas cidades, e transportado até as usinas de reciclagem, onde é realizada uma triagem e separação dos resíduos para britagem em granulometria adequada ao seu determinado uso.

Os fatores preponderantes estão ligados as propriedades do agregado reciclado como sua forma, granulometria, quantidade de finos, porcentagem de material cerâmico, porcentagem de agregado reciclado de concreto e dentre estes, a quantidade de argamassa aderia (argamassa velha) como fator influente na porosidade e durabilidade do concreto (SILVA; MELO, 2020a). Argamassa aderida nada mais é do que restos de argamassa, que no processo de britagem não foi possível descolar ou separar do agregado reciclado.

Estudos realizados por Hansen (1986) retratam que o elo fraco do concreto utilizando agregado reciclado de concreto é a argamassa aderida (argamassa velha). Este resíduo de argamassa que no processo de britagem não foi retirado tem forte efeito sobre as propriedades mecânicas do concreto reciclado com ele produzido.

Outros estudos também realizados em concretos com agregados reciclados de concretos apresentaram uma perda na trabalhabilidade e aumento do consumo de cimento devido à alta absorção da água desse material (MALEŠEV; RADONJANIN; MARINKOVIĆ, 2010). Essa característica ocorre em função do aumento da relação a/c, redução da massa específica, permeabilidade e composição dos agregados reciclados (FROTTÉ et al., 2017), (TENÓRIO et al., 2012).

Pesquisas realizadas por Kirthika e Sinch (2020), Fan, Xiao e Tam (2014), Gomes, Brito e Bravo (2013), Hansen (1986) fazem referência à problemática do uso de agregados reciclados para produção de novos concretos, sendo o "elo fraco" entre agregado reciclado/pasta aderida e argamassa nova. Nesta linha de pesquisa, recorrentes estudos fazem referência ao "elo fraco" no concreto reciclado, associando sempre a argamassa aderida ao agregado reciclado (GOMES; DE BRITO, 2009), (POON; SHUI; LAM, 2004), (TAM; GAO; TAM, 2005).

O agregado de concreto reciclado difere do agregado natural, pois o primeiro contém 
argamassa aderida ao agregado reciclado. A argamassa de cimento aderida ao agregado de concreto reciclado tem maior porosidade $\mathrm{e}$ absorção de água, além de menor resistência do que o agregado natural (SHI et al., 2016). Portanto, apresenta efeitos negativos nas propriedades mecânicas e na durabilidade do concreto fresco e endurecido.

Entretanto, se a argamassa aderida ao agregado reciclado puder ser aprimorada ou eliminada poderá proporcionar melhorias ao concreto reciclado.

A remoção e o reforço da argamassa aderida são os dois principais métodos estudados para melhorar as propriedades do agregado de concreto reciclado (PURUSHOTHAMAN; AMIRTHAVALLI; KARAN, 2015). A primeira forma é a eliminação da argamassa aderida ao agregado reciclado por meio da britagem (GOMES; DE BRITO, 2009), limpeza de água por ultrassom (KATZ, 2004), retificação e aquecimento (ALBAYATI et al., 2016), ou pré-imersão em solução ácida $\mathrm{HCl}, \mathrm{H}_{2} \mathrm{SO}_{4}$ ou $\mathrm{H}_{2} \mathrm{O}_{4}$ (PURUSHOTHAMAN et al., 2015).

Outra técnica é fortalecer a argamassa aderida, revestindo a superfície com solução de polímero, como emulsão de álcool polivinílico (KOU; POON, 2010), solução de silicato de sódio (YANG et al., 2016), e usando as reações entre CO2 e produtos de hidratação (isto é, carbonatação acelerada) para a argamassa aderida (LI et al., 2017), (XUAN; ZHAN; POON, 2016).

O fortalecimento da argamassa aderida ao agregado usando materiais pozolânicos (cinzas volantes, sílica ativa, etc.) é considerado uma técnica eficiente para fortalecer o elo fraco no agregado reciclado, ou seja, a baixa resistência da argamassa velha (SHABAN et al., 2019).

A imersão do agregado reciclado em pasta de pozolana ou a pulverização com materiais pozolânicos (revestindo a sua superfície) faz com que as partículas pozolânicas finas reajam com hidróxido de cálcio $(\mathrm{CH})$ para formar gel de silicato de cálcio hidratado (CASA H), que preenche efetivamente os poros e os espaços vazios ou poros da argamassa velha aderida ao agregado reciclado (HO et al., 2018), (LI; XIAO; ZHOU, 2009). Estudos realizados por (TAM; TAM, 2008) descobriram que a adição de fumaça de sílica na pré-mistura preencheu os vazios da superfície da argamassa aderida e formou uma nova e forte camada ao redor do agregado reciclado de concreto $A R C$.

Vários estudos vêm sendo realizados para melhorar ou mitigar os efeitos negativos que o agregado reciclado exerce nas propriedades do concreto reciclado. E desta forma, viabilizar o uso deste material tão ambulante nos grandes centros urbanos. A possivel melhora de suas propriedades físicas e mecânicas nos possibilita vislumbrar os ganhos ambientais e econômicos da utilização do agregado reciclado em novos produtos para a cadeia produtiva da construção civil.

\subsection{Problemas do agregado reciclado}

Segundo estudos realizados por Cordeeiro et al., (2017), Gomes e De Brito (2009) e Leite (2001), o agregado reciclado apresenta características intrínsecas que afetam o desempenho dos concretos com ele produzidos. O formato irregular do agregado graúdo britado, a menor massa específica e a maior porosidade influenciam diretamente nas propriedades mecânicas dos concretos, tanto no estado fresco, quanto no endurecido.

Nesse sentido, diversos autores como Nagataki et al., (2004), Cordeiro et al., (2017), Gomes e Brito (2009) e Leite (2001) vêm desenvolvendo estudos, principalmente em agregados reciclados de concreto, na tentativa de mitigar os efeitos negativos decorrentes das características desse material, utilizando para isso, tratamentos mecânicos, térmicos e químicos que demandam muitas vezes custo e tempo, mas que levam a resultados promissores.

As escolhas do processo de fragmentação (britagem) são cruciais para estabelecer as condições mais favoráveis e viáveis economicamente para a reciclagem de RCD, e para a utilização do RCD-R na produção de concretos e outros materiais (GOMES et al., 2015).

0 processo de beneficiamento pode também ser uma alternativa para se obterem agregados reciclados com características apropriadas. Estudos demonstraram que a fragmentação de agregados por diferentes processos reduz a porosidade do material, e muitas vezes favorece a obtenção de características similares às do material natural (CORDEIRO et al., 2017).

A fragmentação, por diferentes estágios dos britadores, remove parcialmente a antiga pasta de cimento aderida dos agregados reciclados, reduzindo a porosidade do concreto produzido com agregado reciclado (TAM; GAO; TAM, 2005), (ULSEN et al., 2013).

Estudos realizados por Kong et al., (2010) e Nagataki et al., (2004) têm demonstrado que, tanto a zona de transição ZT entre o agregado reciclado $A R$, quanto a nova matriz de cimento, formam o elo fraco do concreto e afetam as propriedades mecânicas do mesmo. Porém, um 
aperfeiçoamento tecnológico pode ser feito como melhoria no concreto através de um prétratamento do agregado reciclado com solução de sílica ativa, com $10 \%$ em peso (KATZ, 2004).

No entanto, o processo de pré-tratamento antes da produção de concreto, leva a um custo mais alto, limitando assim sua aplicação na construção civil.

\subsection{Agregado reciclado para concreto}

Um abrangente trabalho experimental foi realizado por (HANSEN, 1986; XIAO et al., 2012). Nesse trabalho descobriu-se que, em geral, o procedimento de dosagem de concretos com agregados reciclados não difere muito daquele para o concreto convencional. Contudo, é necessária adição de mais água para atingir uma trabalhabilidade desejada, devido à alta absorção de água do agregado reciclado. Este fator é atribuído à alta concentração de argamassa aderida aos agregados reciclados.

Estudos realizados por (TAM; GAO; TAM, 2005) apresentaram uma técnica de mistura em dois estágios, com o objetivo de melhorar as propriedades físico mecânicas do concreto com agregados reciclados e diminuir a variabilidade de resistência.

Outros pesquisadores como Cordeiro et al., (2017) e Etxeberria et al., (2007) também relataram que os agregados reciclados, que eram usados em condições úmidas, mas não saturados, podiam controlar as propriedades frescas do concreto e a relação efetiva entre a relação água/cimento.

0 fator preponderante da utilização de agregados reciclados em concretos é a elevada absorção de água e o enfraquecimento da zona de transição agregado/pasta de cimento, além da baixa resistência do agregado cerâmico. Segundo a metodologia apontada por Gomes, De Brito (2009), Tam, Gao e Tam (2005) a hidratação da pasta divide-se em duas etapas, sendo a primeira com o objetivo de envolver o agregado, possibilitando a sucção da pasta hidratada aos poros e aberturas do agregado reciclado.

$\mathrm{Na}$ segunda etapa ocorre a cristalização, diminuindo a porosidade do agregado e do concreto como um todo. A segunda adição de água serve para hidratar o resto do cimento necessário para a composição do concreto, minimizando a porosidade da argamassa aderida ao agregado reciclado, elemento influenciador na porosidade do concreto com agregados reciclados.
A Figura 1 demostra os vários fatores que podem influenciar as propriedades mecânicas do concreto com agregado reciclado: argamassa nova, argamassa aderida (argamassa velha), velha zona de transição, nova zona de transição e fissura na zona de transição (nova e velha).

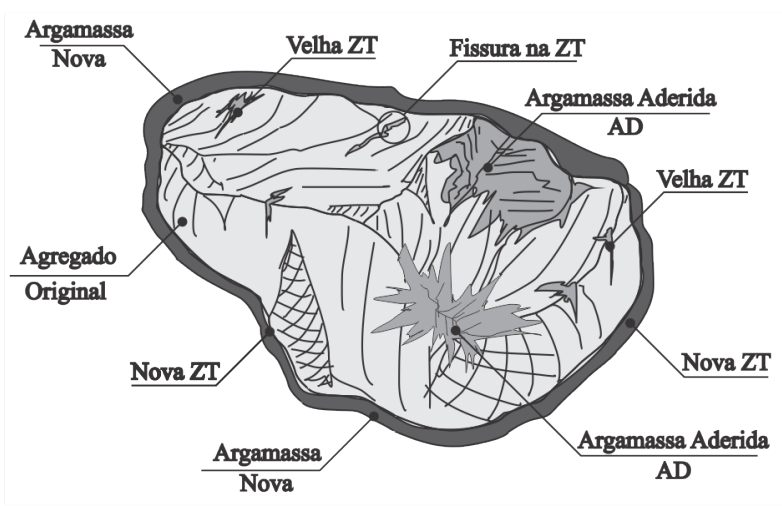

Figura 1. 1 Detalhe do agregado reciclado com argamassa aderida e argamassa nova. Fonte: Acervo dos autores (2021)

Estudos realizados por Cordeiro et al., (2017) apresentam uma proposta de mistura em três etapas. A primeira etapa visa umidificar o agregado graúdo reciclado. A segunda objetiva envolver a superfície do agregado com o cimento em fase de hidratação, tal qual a proposta de Gomes, De Brito (2009), Tam, Gao e Tam (2005), Tam e Tam (2008), além de garantir que o agregado reciclado absorva a água da mistura. Já a terceira e última etapa, na qual é adicionado o restante dos materiais necessários para a produção do concreto, visa homogeneizar a mistura até atingir a trabalhabilidade desejada.

Ulsen et al., (2013) confirmou que mais estágios de britagem resultam em uma redução da absorção de água do agregado graúdo reciclado em aproximadamente $10 \%$ a $20 \%$, comparados com o uso de uma e duas etapas de britagem. Já em uma terceira etapa, utilizando um britador de eixo vertical, obteve-se uma redução de $40 \%$ a $60 \%$ na absorção de água do agregado reciclado.

Estudos sobre o concreto reciclado, indicaram que a zona de transição ZT é o elo mais fraco no concreto, interface entre a pasta de cimento e a AR (NAGATAKI et al., 2004). Portanto, a modificação da microestrutura na ZT tem sido uma das grandes preocupações para melhorar as propriedades do agregado reciclado de concreto (KONG et al., 2010).

Estudos sobre o processo de britagem demostram que este tratamento pode aumentar em torno de $15 \%$ a $30 \%$, a resistência à compressão em idades mais avançadas. 
Argamassa aderida ao agregado reciclado é basicamente massa de concreto velha, que no processo de britagem não foi possível ser retirada totalmente. Ou seja, é a argamassa que envolvia o agregado graúdo, na microestrutura do concreto antigo. Muitas das vezes, ficam crostas ou camadas de argamassa acopladas ao agregado, o que provoca baixo desempenho do concreto, quando utiliza-se o agregado reciclado em sua composição.

Em um estudo realizado por Bairagi, Vidyadhara e Ravande (1990) para dosagem de concreto reciclado, o agregado reciclado foi produzido a partir do concreto, sendo dosado para uma determinada resistência, moldados e curados por 28 dias, submerso em água e britado aos três meses de idade, usando um britador de mandíbulas em laboratório. Após a britagem, o agregado foi caracterizado e comparado ao agregado natural original. A Tabela 1 apresenta a comparação das propriedades dos agregados.

TABELA 1. Propriedades do agregado natural e reciclado. Fonte: (BAIRAGI; VIDYADHARA; RAVANDE, 1990) adaptado

\begin{tabular}{crcc}
\hline $\begin{array}{c}\text { Tipo de } \\
\text { Agregado }\end{array}$ & $\begin{array}{c}\text { Massa } \\
\text { Especifica } \\
\left(\mathrm{Kg} / \mathbf{m}^{3}\right)\end{array}$ & $\begin{array}{c}\text { Absorção } \\
\text { de Água } \\
(\%)\end{array}$ & $\begin{array}{c}\text { Modulo } \\
\text { de Finura }\end{array}$ \\
\hline $\begin{array}{c}\text { Agregado } \\
\text { Natural }\end{array}$ & 2,90 & 2,00 & 6,45 \\
\hline $\begin{array}{c}\text { Agregado } \\
\text { Reciclado }\end{array}$ & 2,56 & 5,76 & 6,57 \\
\hline
\end{tabular}

Este estudo demonstra a variabilidade das propriedades físicas de um mesmo material, no estado de agregado natural e no estado de agregado reciclado. 0 estudo da composição resultante da mistura entre esses dois materiais é de suma importância para a qualidade dos futuros concretos com eles produzidos.

\section{CONCRETO RECICLADO}

Inúmeros estudos estão sendo realizados com intuito de fomentar o uso do RCD como agregado reciclado no concreto. Com isso, as pesquisas variam em quantidades de substituição e tipo de agregado reciclado.

Os agregados reciclados podem ser classificados como ARC e ARM, sendo que estudos abordando o uso do ARC no concreto reciclado são mais facilmente encontrados na literatura.

0 agregado reciclado misto é dado como o mais problemático, devido à alta quantidade de impurezas presentes no agregado, tais como torrões de argamassa, cerâmica vermelha e outros materiais.

Os estudos de Bairagi, Vidyadhara e Ravande (1990) demonstram o aumento da capacidade de absorção do agregado reciclado de concreto.
Estes resultados mostram que sua capacidade pode triplicar em relação ao agregado natural.

A Tabela 2 apresenta a quantidade dos teores de substituição de agregado graúdo natural por agregado graúdo reciclado, estudados por diferentes pesquisadores.

Podemos observar que as quantidades de substituições variaram entre os diferentes pesquisadores. Um breve relato das pesquisas com o teor de substituição de agregado graúdo natural por agregado graúdo reciclado pode ser observado na Tabela 2.

TABELA 2. Porcentagem de substituição do agregado graúdo reciclados como substituição ao natural por diferentes pesquisadores. Fonte: (VERIAN; ASHRAF; CAO, 2018) adaptado.

\begin{tabular}{|c|c|}
\hline Referências & $\begin{array}{l}\text { Agregado graúdo } \\
\text { reciclado }\end{array}$ \\
\hline (LEITE, 2001) & $\begin{array}{c}0 \%, 11,5 \%, 50, \%, \\
88,5 \%, 100 \%\end{array}$ \\
\hline $\begin{array}{l}\text { (GÓMEZ-SOBERÓN, } \\
\text { 2002) }\end{array}$ & $0 \%$ e $100 \%$ \\
\hline $\begin{array}{l}\text { (OLORUNSOGO; } \\
\text { PADAYACHEE, 2002) }\end{array}$ & $0 \%, 50 \%$ e $100 \%$ \\
\hline (ZAHARIEVA et al., 2003) & $0 \%$ e $100 \%$ \\
\hline (POON et al., 2004) & $\begin{array}{c}0 \%, 20 \%, 50 \% \text { e } \\
100 \%\end{array}$ \\
\hline $\begin{array}{l}\text { (DE BRITO; PEREIRA; } \\
\text { CORREIA, 2005) }\end{array}$ & 33,66 e $100 \%$ \\
\hline (XIAO; LI; ZHANG, 2005) & $\begin{array}{c}0 \%, 30 \%, 50 \% \\
70 \%, 100 \%\end{array}$ \\
\hline $\begin{array}{l}\text { (STURTEVANT; COX; } \\
\text { AVE, 2006) }\end{array}$ & $\begin{array}{c}0 \%, 20 \%, 50 \% \\
100 \%\end{array}$ \\
\hline $\begin{array}{l}\text { (ETXEBERRIA et al., } \\
2007)\end{array}$ & $\begin{array}{c}0 \%, 20 \%, 50 \% \text { e } \\
100 \%\end{array}$ \\
\hline (TAM; TAM, 2007) & $0 \%, 20 \%$ e $100 \%$ \\
\hline (RAHAL, 2007) & $0 \%$ e $100 \%$ \\
\hline $\begin{array}{l}\text { (KOU; POON; DIXON, } \\
\text { 2007) }\end{array}$ & $\begin{array}{c}0 \%, 20 \%, 50 \% \text { e } \\
100 \%\end{array}$ \\
\hline (CABRAL et al., 2008) & $\begin{array}{c}0 \%, 25 \%, 33 \% \\
50 \%\end{array}$ \\
\hline (LIU; CHEN, 2008) & $0 \%$ e $100 \%$ \\
\hline $\begin{array}{l}\text { (CORINALDESI; } \\
\text { MORICONI, 2009) }\end{array}$ & $0 \%$ e $100 \%$ \\
\hline (LINTZ et al., 2012) & $\begin{array}{c}0 \%, 20 \%, 50 \% \\
80 \% \text { e } 100 \%\end{array}$ \\
\hline (VERIAN et al., 2013) & $\begin{array}{c}0 \%, 30 \%, 50 \% \text { e } \\
100 \%\end{array}$ \\
\hline $\begin{array}{l}\text { (BUTLER; WEST; TIGHE, } \\
\text { 2013) }\end{array}$ & $0 \%$ e $100 \%$ \\
\hline $\begin{array}{l}\text { (GOMES; DE BRITO; } \\
\text { BRAVO, 2013) }\end{array}$ & $\begin{array}{c}6,25,12,5,25 \mathrm{e} \\
50 \%\end{array}$ \\
\hline $\begin{array}{l}\text { (LANDOLFO; OLIVEIRA; } \\
\text { NOGUEIRA, 2014) }\end{array}$ & $50 \%$ e $100 \%$ \\
\hline $\begin{array}{l}\text { (ALIABDO; ABD- } \\
\text { ELMOATY; HASSAN, } \\
\text { 2014) }\end{array}$ & $\begin{array}{l}0 \%, 25 \%, 50 \% \\
75 \%, 100 \%\end{array}$ \\
\hline
\end{tabular}




\begin{tabular}{lc}
$\begin{array}{lc}\text { (AIT MOHAMED AMER et } \\
\text { al., 2016) }\end{array}$ & $\begin{array}{c}0 \%, 20 \%, 40 \%, \\
60 \%, 80 \%, 100 \%\end{array}$ \\
\hline $\begin{array}{l}\text { (AFROUGHSABET; } \\
\text { BIOLZI; } \\
\text { OZBAKKALOGLU, 2017) }\end{array}$ & $0 \%, 50 \%$ e $100 \%$ \\
\hline $\begin{array}{l}\text { (GAO; ZHANG; NOKKEN, } \\
\text { 2017) }\end{array}$ & $\begin{array}{c}0 \%, 30 \%, 50 \% \text { e } \\
100 \%\end{array}$ \\
\hline $\begin{array}{l}\text { (KATKHUDA; } \\
\text { SHATARAT, 2017) }\end{array}$ & $0 \%$ e $20 \%$ \\
\hline $\begin{array}{l}\text { (KURDA; DE BRITO; } \\
\text { SILVESTRE, 2017) }\end{array}$ & $0 \%$ e $100 \%$ \\
\hline $\begin{array}{l}\text { (PEDRO; DE BRITO; } \\
\text { EVANGELISTA, 2017) }\end{array}$ & $0 \%, 50 \%$ e $100 \%$ \\
\hline $\begin{array}{l}\text { (STROHER et al., 2017) } \\
\text { (SMITH, 2018) apud }\end{array}$ & $0 \%, 5 \%, 25 \%$ e \\
(VERIAN; ASHRAF; CAO, & $0 \%, 15 \%, 30 \%$ e \\
2018) & $50 \%$ \\
\hline $\begin{array}{l}\text { (FERNANDES; MELO, } \\
\text { 2020) }\end{array}$ & $15 \%$ e 30\% \\
\hline
\end{tabular}

As pesquisas analisadas apresentam uma concentração nos percentuais de substituição de $20 \%, 25 \%, 30 \%$ e $50 \%$, demostrando um cenário favorável ao uso do agregado reciclado. Entretanto, a falta de normatização para o uso desse tipo de agregado limita a possibilidade de seu uso em concretos estruturais.

Os estudos de Poon et al., (2004), Evangelista, De Brito (2007), Gesoglu et al., (2015), Kurad et al., (2017) demonstram que a resistência do concreto com agregado reciclado pode ser maior que a do concreto com agregado natural, principalmente em idades mais avançadas. Associa-se estes resultados à argamassa aderida, nos quais a $A D$ contém aglomerante não hidratado aderido às superfícies das partículas do agregado reciclado, que reagem com água, aumentando a resistência do concreto.

Outros estudos (VERIAN; ASHRAF; CAO, 2018) demonstram que dependendo do nível de umidade, a resistência à compressão pode ser reduzida em até $30 \%$ ou aumentar em até $20 \%$ para $100 \%$ de agregados substituídos por agregado reciclado.

Atribui-se esse fenômeno de menor resistência mecânica do concreto produzido com agregado reciclado, à presença de dois tipos de zonas de transição interfacial (ZTI) na matriz. Esta ZTI representa a ligação entre o agregado e a pasta aderida ao agregado, sendo que a ZTI é normalmente mais fraca que a pasta de cimento hidratada. Tam, Gao e Tam (2005), Tam, e Tam (2008), Kong et al., (2010), Kou, Poon e Agrela (2011), Lotfi et al., (2014).

Estudos de Dilbas, Şimsek e Çakir (2014), em corpos de prova, estabeleceram em $30 \%$ a proporção ótima para utilização de agregados reciclados. Já em outros estudos, como o de Gomes e Brito (2009), relata-se que é tecnicamente viável substituir parte do agregado graúdo natural por agregado reciclado de concreto até uma fração de $50 \%$ do volume em massa na produção do concreto, e uma fração de $25 \%$ do agregado natural por agregados cerâmicos, sem que estes afetem negativamente as propriedades mecânicas do concreto.

Estudos realizados por Silva et al., (2015) demonstram em seus experimentos que é viável tecnicamente a substituição parcial de agregados naturais por reciclados em fração < $50 \%$ em massa para produção de concretos. Estes estudos apresentaram resistências compatíveis a concretos convencionais.

\subsection{Resistência do concreto reciclado}

Estudos realizados por Juan-Valdés (2018) em um comparativo entre concreto reciclado com $50 \%$ de substituição e o convencional, a substituição do agregado natural por agregado reciclado causou uma redução de $25 \%$ da resistência à compressão no concreto reciclado em comparação ao concreto convencional, ambos dosados para atingir uma resistência de $40 \mathrm{MPa}$.

Gonzalez; Etxeberria (2014) observaram um declínio semelhante na resistência à compressão aos 28 dias, com uma redução de $26 \%$ na resistência do concreto produzido e uma substituição de $50 \%$ de agregado natural por ARM, contendo $67,3 \%$ de materiais cerâmicos, $22,2 \%$ de produtos de concreto, $9,8 \%$ de agregados não ligados e $0,8 \%$ de impurezas.

Os resultados dos estudos apresentados por JuanValdés (2018), Verian, Ashraf e Cao (2018) e Gonzalez; Etxeberria (2014) demonstram o potencial que o agregado reciclado tem como agregado para concreto e materiais na construção.

Conforme estudos demonstram, o agregado reciclado possui grande potencial de uso, e os avanços tecnológicos favorecem ainda mais a possibilidade de seu uso na construção civil.

\section{DISCUSSÕES}

Os agregados reciclados provenientes de estruturas de concreto, pré-moldado e artefatos de concreto são classificados como ARC. Estes são mais requisitados quando usados em concreto e apresentam melhores desempenhos em relação ao ARM.

O ARM apresenta grande quantidade de material cerâmico proveniente de alvenarias e telhas cerâmicas, o que provoca baixa resistência nos concretos com eles produzidos. 
0 custo do $\mathrm{m}^{3}$ do ARM é, em média, $1 / 3$ do agregado natural britado, o que favorece o seu uso e fomenta pesquisas com o objetivo de alcançar melhorias nas propriedades deste material.

Pesquisas apontam que a alta absorção do agregado reciclado pode ser mitigada, melhorando as propriedades do concreto, tanto no estado fresco, como no estado endurecido.

Estas pesquisas também demonstraram que é possível modificar ou até mesmo controlar certas propriedades físicas e mecânicas desejáveis deste material, proporcionando melhores resultados nos concretos com eles produzidos. Porém, esta melhoria no material é atrelada a um custo relativamente alto, devido aos mecanismos utilizados.

Uma redução em média de $25 \%$ na resistência do concreto reciclado, com $50 \%$ de substituição, pode ser observada em pesquisas, e isso pode variar com a quantidade de agregado reciclado em massa no concreto.

Estudos indicam que o uso do ARC e ARM podem ser utilizados em concretos. Entretanto, é de conhecimento dos pesquisadores que sua substituição provoca uma redução em suas propriedades mecânicas, como resistência e durabilidade. Contudo, com um controle adequado, é possível planejar o uso do concreto reciclado para diversas finalidades como mobiliário urbano, calçadas, blocos e pisos, e até mesmo em concretos estruturais.

\section{CONCLUSÕES}

O fator preponderante para uso desse material é o elo fraco entre agregado reciclado, argamassa velha e argamassa nova. Este elo fraco é provocado pela alta porosidade das partículas recicladas, e por essa argamassa aderida que pode ser eliminada ou reduzida na fase de beneficiamento do agregado reciclado.

Diversos estudos têm sido desenvolvidos com o objetivo de fortalecer ou eliminar o "elo fraco" do concreto reciclado, de modo a reduzir a alta porosidade deste. Assim, torna-se indispensável aprimorar tecnicamente o referencial teórico científico, com base em estudos experimentais do uso deste material tão promissor, com um custo baixo e com elevado ganho ambiental.

Este estudo contribui para um alcance em parâmetros de qualidade, para produção e uso dos agregados reciclados em concretos. As descobertas dessas pesquisas retratam um panorama favorável à utilização desse resíduo como agregado em concretos ou materiais construtivos, que possam ser introduzidos na cadeia produtiva da construção civil.

A qualidade do concreto reciclado está intimamente relacionada com as propriedades físicas e mecânicas do agregado reciclado. Aliado à possibilidade de controlar essas propriedades, estimula-se pesquisas e torna-se possivel o alcance de um produto com melhor qualidade e a um menor custo, permitindo um maior controle tecnológico do concreto com eles produzidos.

Contudo, a falta de normas técnicas que regulamentam o seu uso no Brasil, limitam a sua utilização, permitindo a sua introdução apenas em concretos não estruturais como calçadas, contrapiso, blocos de vedação e outros. Mas de certa forma, essa limitação fomenta possíveis cenários a longo e curto prazo para a viabilidade do seu uso em concretos estruturais, que pesquisas científicas devem abordar.

\section{REFERÊNCIAS}

AFROUGHSABET, V.; BIOLZI, L.; OZBAKKALOGLU, $T$. Influence of double hooked-end steel fibers and slag on mechanical and durability properties of high performance recycled aggregate concrete. Composite Structures, v. 181, p. 273284, 1 dez. 2017.

AIT MOHAMED AMER, A. et al. Rheological and mechanical behavior of concrete made with presaturated and dried recycled concrete aggregates. Construction and Building Materials, v. 123, p. 300-308, 1 out. 2016.

AL-BAYATI, H. K. A. et al. Evaluation of various treatment methods for enhancing the physical and morphological properties of coarse recycled concrete aggregate. Construction and Building Materials, v. 112, p. 284-298, 1 jun. 2016.

ALIABDO, A. A.; ABD-ELMOATY, A. E. M.; HASSAN, H. H. Utilization of crushed clay brick in concrete industry. Alexandria Engineering Journal, v. 53, n. 1, p. 151-168, mar. 2014.

ALMEIDA, S. L. M. DE; LUZ, A. B. DA. Manual de Agregados para Construção Civil. Rio de Janeiro, 2009.

ALMEIDA, S. P. DE. Uso De Politereftalato De Etileno (Pet) Como Agregado Em Peças De Concreto Para Pavimento Intertravado. [S.l.] Universidade Federal de Campina Grande, 2016.

ALVES, A. V. et al. Mechanical properties of structural concrete with fine recycled ceramic aggregates. Construction and Building Materials, v. 64, p. 103-113, 14 ago. 2014. 
AMORIM, P.; BRITO, J. DE. Concrete Made With Coarse Concrete Aggregate: ACl Materials Journal, v. 109, n. March, p. 195-204, 2012.

BAIRAGI, N. K.; VIDYADHARA, H. S.; RAVANDE, K. Mix design procedure for recycled aggregate concrete. Construction and Building Materials, v. 4, n. 4, p. 188-193, 1990.

BASTOS, I. A.; CRUZ, L. F. DA; WOELFFEL, A. B. Fabricação De Blocos De Concreto Para Vedação Com $O$ Uso De Agregados Reciclados Em Canteiro De Obras. Revista Científica Faesa, v. 12, n. 1, p. 52-58, 2016.

BRASIL. Política Nacional de Resíduos Sólidos. Diário Oficial da União, p. 1-21, 2010.

BRASILEIRO, L. L.; MATOS, J. M. E. Revisão bibliográfica: reutilização de resíduos da construção e demolição na indústria da construção civil. Cerâmica, v. 61 , n. 358 , p. 178189, 2015.

BRAVO, M. et al. Durability performance of concrete with recycled aggregates from construction and demolition waste plants. Construction and Building Materials, v. 77, p. 357-369, 2015.

BUTLER, L.; WEST, J. S.; TIGHE, S. L. Effect of recycled concrete coarse aggregate from multiple sources on the hardened properties of concrete with equivalent compressive strength. Construction and Building Materials, v. 47, p. 1292-1301, 2013.

CABRAL, A. E. B. et al. Influence of the type of recycled aggregate from construction and demolition waste on the module of deformation of recycled aggregate concrete. RIEM - IBRACON Structures and Materials Journal, v. 1, n. 2, p. 171-181, 2008.

CONAMA. CONAMA 307:2002 - Gestão de Resíduos Sólidos (Construção Civil), 2002.

CORDEIRO, L. DE N. P. et al. Avaliação de processos de misturas de concretos com agregados graúdos reciclados. Ambiente Construído, v. 17, n. 3 , p. $255-265,30$ jun. 2017.

CORINALDESI, V.; MORICONI, G. Influence of mineral additions on the performance of $100 \%$ recycled aggregate concrete. Construction and Building Materials, v. 23 , n. 8 , p. 2869-2876, ago. 2009.

DE BRITO, J.; PEREIRA, A. S.; CORREIA, J. R. Mechanical behaviour of non-structural concrete made with recycled ceramic aggregates. Cement and Concrete Composites, v. 27, n. 4, p. 429433, abr. 2005.
DILBAS, H.; ŞIMŞEK, M.; ÇAKIR, Ö. An investigation on mechanical and physical properties of recycled aggregate concrete (RAC) with and without silica fume. Construction and Building Materials, v. 61, n. March 2006, p. 5059, 2014.

ETXEBERRIA, M. et al. Influence of amount of recycled coarse aggregates and production process on properties of recycled aggregate concrete. Cement and Concrete Research, v. 37, n. 5, p. 735-742, maio 2007.

EVANGELISTA, L.; DE BRITO, J. Mechanical behaviour of concrete made with fine recycled concrete aggregates. Cement and Concrete Composites, v. 29, n. 5, p. 397-401, maio 2007.

FAN, Y.; XIAO, J.; TAM, V. W. Y. Effect of old attached mortar on the creep of recycled aggregate concrete. Structural Concrete, v. 15, n. 2, p. 169-178, 2014.

FERNANDES, G. D. S.; MELO, C. E. L. DE. Estudo das características físicas e hidráulicas de concretos permeáveis com RCD. Paranoá: cadernos de arquitetura e urbanismo, n. 26, p. 205-218, 28 maio 2020.

FROTTÉ, C. et al. Estudo das propriedades físicas e mecânicas de concreto com substituição parcial de agregado natural por agregado reciclado proveniente de RCD. Revista Materia, v. 22, n. 2, 2017.

GAO, D.; ZHANG, L.; NOKKEN, M. Mechanical behavior of recycled coarse aggregate concrete reinforced with steel fibers under direct shear. Cement and Concrete Composites, v. 79, p. 18, 1 maio 2017.

GESOGLU, M. et al. Failure characteristics of self-compacting concretes made with recycled aggregates. Construction and Building Materials, v. 98, p. 334-344, 2015.

GOMES, M.; DE BRITO, J. Structural concrete with incorporation of coarse recycled concrete and ceramic aggregates. Portugal Sb07 Sustainable Construction, Materials and Practices: Challenge of the Industry for the New Millennium, Pts 1 and 2, p. 887-894, 2007.

GOMES, M.; DE BRITO, J. Structural concrete with incorporation of coarse recycled concrete and ceramic aggregates: Durability performance. Materials and Structures/Materiaux et Constructions, v. 42, n. 5, p. 663-675, jun. 2009.

GOMES, M.; DE BRITO, J.; BRAVO, M. Mechanical Performance of Structural Concrete with the Incorporation of Coarse Recycled Concrete and Ceramic Aggregates. Journal of Materials in 
Civil Engineering, v. 26, n. 10, p. 04014076, 6 nov. 2013.

GOMES, P. C. C. et al. Comminution and sizing processes of concrete block waste as recycled aggregates. Waste Management, v. 45, p. 171179, 26 jan. 2015.

GÓMEZ-SOBERÓN, J. M. V. Porosity of recycled concrete with substitution of recycled concrete aggregate: An experimental study. Cement and Concrete Research, v. 32, n. 8, p. 1301-1311, 2002.

GONZALEZ-COROMINAS, A.; ETXEBERRIA, M. Properties of high performance concrete made with recycled fine ceramic and coarse mixed aggregates. Construction and Building Materials, v. 68, p. 618-626, 15 out. 2014.

HANSEN, T. C. Recycled aggregates and recycled aggregate concrete second state-of-the-art report developments 1945-1985. Materials and Structures, v. 19, n. 3, p. 201-246, 1986.

HO, H. L. et al. Pore-structures and durability of concrete containing pre-coated fine recycled mixed aggregates using pozzolan and polyvinyl alcohol materials. Construction and Building Materials, v. 160, p. 278-292, 30 jan. 2018.

JUAN-VALDÉS, A. et al. Mechanical and microstructural characterization of nonstructural precast concrete made with recycled mixed ceramic aggregates from construction and demolition wastes. Journal of Cleaner Production, v. 180, p. 482-493, 2018.

KATKHUDA, H.; SHATARAT, N. Improving the mechanical properties of recycled concrete aggregate using chopped basalt fibers and acid treatment. Construction and Building Materials, v. 140, p. 328-335, 1 jun. 2017.

KATZ, A. Treatments for the improvement of recycled aggregate. Journal of Materials in Civil Engineering, v. 16, n. 6, p. 597-603, nov. 2004.

KIRTHIKA, S. K.; SINGH, S. K. Durability studies on recycled fine aggregate concrete. Construction and Building Materials, v. 250, p. 118850, jul. 2020.

KLEIN, F. B.; GONÇALVES DIAS, S. L. F. A deposição irregular de resíduos da construção civil no município de São Paulo: um estudo a partir dos instrumentos de políticas públicas ambientais. Desenvolvimento e Meio Ambiente, v. 40, p. 483-506, 30 abr. 2017.

KONG, D. et al. Effect and mechanism of surface-coating pozzalanics materials around aggregate on properties and ITZ microstructure of recycled aggregate concrete. Construction and Building Materials, v. 24, n. 5, p. 701-708, 2010.

KOU, S. C.; POON, C. S. Properties of concrete prepared with PVA-impregnated recycled concrete aggregates. Cement and Concrete Composites, v. 32, n. 8, p. 649-654, set. 2010.

KOU, S. C.; POON, C. S.; AGRELA, F. Comparisons of natural and recycled aggregate concretes prepared with the addition of different mineral admixtures. Cement and Concrete Composites, v. 33, n. 8, p. 788-795, 2011.

KOU, S. C.; POON, C. S.; DIXON, C. Influence of fly ash as cement replacement on the properties of recycled aggregate concrete. Journal of Materials in Civil Engineering, v. 19, n. 9, p. 709-717, 2007.

KURAD, R. et al. Effect of incorporation of high volume of recycled concrete aggregates and fly ash on the strength and global warming potential of concrete. Journal of Cleaner Production, v. 166, p. 485-502, 2017.

KURDA, R.; DE BRITO, J.; SILVESTRE, J. D. Influence of recycled aggregates and high contents of fly ash on concrete fresh properties. Cement and Concrete Composites, v. 84, p. 198-213, 1 nov. 2017.

LANDOLFO, R.; OLIVEIRA, M. P. DE; NOGUEIRA, N. A. DE S. Estudo Comparativo da Utilização de Resíduo Cerâmico, como Agregado Graúdo, na Produção de Concreto. Cerâmica Industrial, v. 19, n. 5, p. 35-41, 2014.

LEITE, M. B. Avaliação de Propriedades Mecânicas de Concreto Produzidos com Agregados Reciclados de Resíduos de Construção. [s.l.] Universidade Federal do Rio Grande do Sul, mar. 2001.

LI, J.; XIAO, H.; ZHOU, Y. Influence of coating recycled aggregate surface with pozzolanic powder on properties of recycled aggregate concrete. Construction and Building Materials, v. 23, n. 3, p. 1287-1291, mar. 2009.

LI, L. et al. Effect of carbonated recycled coarse aggregate on the dynamic compressive behavior of recycled aggregate concrete. Construction and Building Materials, v. 151, p. 52-62, 1 out. 2017.

LINTZ, R. C. C. et al. Study of the reuse of construction residues in concrete employed by blocks manufacture. Revista IBRACON de Estruturas e Materiais, v. 5, n. 2, p. 166-181, abr. 2012.

LIU, J.; CHEN, B. Mechanical properties of high strength concrete with field-demolished concrete 
as aggregates. Journal of ASTM International, v. 5, n. 10, 2008.

LOTFI, S. et al. Mechanical recycling of EOL concrete into high-grade aggregates. Resources, Conservation and Recycling, v. 87, p. 117-125, 2014.

MALEŠEV, M.; RADONJANIN, V.; MARINKOVIĆ, S. Recycled concrete as aggregate for structural concrete production. Sustainability, v. 2, n. 5, p. 1204-1225, 2010.

MARTINS, D. J.; CORREIA, J. R.; DE BRITO, J. The effect of high temperature on the residual mechanical performance of concrete made with recycled ceramic coarse aggregates. Fire and Materials, v. 40, n. 2, p. 289-304, 1 mar. 2016.

MEHTA, P. K.; MONTEIRO, P. J. M. Concreto: estrutura, propriedades e materiais. In: $3 a$ ed. ed. São Paulo - SP: Editora Pini, 2008. p. 573.

METSO. Mercado de agregados mostra reinvenção diante da crise. Disponível em: <https://www.metso.com/br/brasil/noticias/20 19/mercado-de-agregados-mostra-reinvencaodiante-da-crise/>.

NAGATAKI, S. et al. Assessment of recycling process induced damage sensitivity of recycled concrete aggregates. Cement and Concrete Research, v. 34, n. 6, p. 965-971, jun. 2004.

OLORUNSOGO, F. T.; PADAYACHEE, N. Performance of recycled aggregate concrete monitored by durability indexes. Cement and Concrete Research, v. 32, n. 2, p. 179-185, 2002.

PEDRO, D.; DE BRITO, J.; EVANGELISTA, L. Evaluation of high-performance concrete with recycled aggregates: Use of densified silica fume as cement replacement. Construction and Building Materials, v. 147, p. 803-814, 30 ago. 2017.

POON, C. S. et al. Influence of moisture states of natural and recycled aggregates on the slump and compressive strength of concrete. Cement and Concrete Research, v. 34, n. 1, p. 31-36, jan. 2004.

POON, C. S.; SHUI, Z. H.; LAM, L. Effect of microstructure of ITZ on compressive strength of concrete prepared with recycled aggregates. Construction and Building Materials, v. 18, n. 6, p. 461-468, 2004.

PURUSHOTHAMAN, R.; AMIRTHAVALLI, R. R.; KARAN, L. Influence of Treatment Methods on the Strength and Performance Characteristics of Recycled Aggregate Concrete. Journal of Materials in Civil Engineering, v. 27, n. 5, p. 04014168, 1 maio 2015.
RAHAL, K. Mechanical properties of concrete with recycled coarse aggregate. Building and Environment, v. 42, n. 1, p. 407-415, jan. 2007.

SACHO, S. D. et al. Avaliação Econômica E De Emissões De Co2 Da Reciclagem De Resíduos De Construção E Demolição Classe a: Estudo De Caso Para Goiânia - Go. MIX Sustentável, v. 2, n. 2, p. 20, 2016.

SANTANA, V. M. DE et al. Utilização de concreto reciclado na aplicação de elementos estruturais. Anais do XV Encontro Latino Americano de Iniciação Científica e XI Encontro Latino Americano de Pós-Graduação, p. 1-6, 2011.

SHABAN, W. M. et al. Quality improvement techniques for recycled concrete aggregate: A review. Journal of Advanced Concrete Technology, v. 17, n. 4, p. 151-167, 2019.

SHI, C. et al. Performance enhancement of recycled concrete aggregate - A review Journal of Cleaner Production Elsevier Ltd, 20 jan. 2016.

SILVA, D. A.; LUNA DE MELO, C. E. Processo de britagem e sua influência nas características físicas do agregado graúdo para concreto. Paranoá: cadernos de arquitetura e urbanismo, v. 26, n. 26, p. 36-47, 28 maio 2020.

SILVA, D. DE A. E. Estudo da influência do índice de forma do graúdo nas propriedades mecânicas do concreto. [s.l.] Dissertação de (Mestrado) - Universidade Federal de Goiás, Escola de Engenharia Civil, 2012.

SILVA, D. DE A. E; MELO, C. E. L. DE. Utilização de material cerâmico proveniente do RCD para aplicação em concreto: uma revisão. Risco Revista de Pesquisa em Arquitetura e Urbanismo (Online), v. 19, p. 1-15, 2021.

SILVA, R. B. DA et al. Concretos secos produzidos com agregados reciclados de RCD separados por densidade. Ambiente Construído, v. 15, n. 4, p. 335-349, dez. 2015.

SILVA, R. V.; DE BRITO, J.; DHIR, R. K. Properties and composition of recycled aggregates from construction and demolition waste suitable for concrete production. Construction and Building Materials, v. 65, p. 201-217, 29 ago. 2014.

STROHER, A. P. et al. Utilização da Cerâmica de Entulho na Substituição de Agregado Graúdo do Concreto. Cerâmica Industrial, v. 22, n. 4, p. 34-46, 2017.

TAM, V. W. Y.; TAM, C. M. Assessment of durability of recycled aggregate concrete produced by two-stage mixing approach. Journal of Materials Science. Anais...maio 2007 
TAM, V. W. Y.; TAM, C. M. Diversifying two-stage mixing approach (TSMA) for recycled aggregate concrete: TSMAs and TSMAsC. Construction and Building Materials, v. 22, n. 10, p. 2068-2077, out. 2008.

TENÓRIO, J. J. L. et al. Concrete produced with recycled aggregates. Revista IBRACON de Estruturas e Materiais, v. 5, n. 5, p. 692-701, out. 2012.

TROCA, J. R. Estimativa da geração e destinação do resíduo da construção civil na cidade de Lavras - MG. [s.l.] Universidade Federal de Lavras, 2007.

ULSEN, C. et al. Production of recycled sand from construction and demolition waste. Construction and Building Materials, v. 40, p. 1168-1173, 2013.

VERIAN, K. et al. Using Recycled Concrete as Aggregate in Concrete Pavements to Reduce Materials CostJoint Transportation Research Program, 31 dez. 2013. Disponível em: <http://docs.lib.purdue.edu/jtrp/1539/>

VERIAN, K. P.; ASHRAF, W.; CAO, Y. Properties of recycled concrete aggregate and their influence in new concrete production. Resources, Conservation and Recycling, v. 133, p. 30-49, 1 jun. 2018.

$\mathrm{XIAO}$, J. et al. An overview of study on recycled aggregate concrete in China (1996-2011). Construction and Building Materials, v. 31, p. 364-383, 2012.

XIAO, J.; LI, J.; ZHANG, C. Mechanical properties of recycled aggregate concrete under uniaxial loading. Cement and Concrete Research, v. 35, n. 6, p. 1187-1194, jun. 2005.

XUAN, D.; ZHAN, B.; POON, C. S. Assessment of mechanical properties of concrete incorporating carbonated recycled concrete aggregates. Cement and Concrete Composites, v. 65, p. 6774, 1 jan. 2016.

YANG, L.-X. et al. Effects of Recycled Coarse Aggregate Reinforcing Treated by Water-Glass on the Performance of Recycled Concrete. Proceedings of the 3rd International Conference on Material Engineering and Application (ICMEA 2016). Anais...Paris, France: Atlantis Press, 2016Disponível em: <http://www.atlantispress.com/php/paper-details.php?id=25866564>

ZAHARIEVA, R. et al. Assessment of the surface permeation properties of recycled aggregate concrete. Cement and Concrete Composites, v. 25, n. 2, p. 223-232, 2003.

\section{AUTORES}

ORCID: https: //orcid.org/0000-0001-6402-8086

DANILLO DE ALMEIDA E SILVA (DAS), MSc.| Universidade de Brasília | Faculdade de Arquitetura e Urbanismo | Brasília (DF) - Brasil | Campus Universitário Darcy Ribeiro. Asa Norte. Brasília-DF, 70842-970 | e-mail: arq.danillo@gmail.com

ORCID: https: / / orcid.org/0000-0003-2160-6776 CARLOS EDUARDO LUNA DE MELO (CELM), Dr. I Universidade de Brasília | Faculdade de Arquitetura e Urbanismo | Brasília (DF) - Brasil | Campus Universitário Darcy Ribeiro. Asa Norte. Brasília-DF, 70842-970 | E-mail: carlosluna@unb.br

\section{HOW TO CITE THIS ARTICLE}

SILVA, Danilo de Almeida e; MELO, Carlos Eduardo Luna de. Argamassa Aderida, o Elo Fraco no Concreto Reciclado: Uma Revisão. MIX Sustentável, v. 8, n. 1, p. 78-89, jan. 2022. ISSN 24473073.Disponível em: <http://www.nexos.ufsc.br/index.php/mixsuste ntavel>. DOI: http://dx.doi.org/10.29183/24473073.MIX2022.v8.n1.78-89

Submitted: 04/03/2021

Approved: $11 / 08 / 2021$

Published: $01 / 12 / 2021$

Editor responsável: Paulo Cesar Machado Ferroli

Registro da contribuição de autoria:

Taxonomia CRediT (http://credit.niso.org/)

DAS; CELM: análise formal, metodologia, administração do projeto, validação, escrita revisão e edição.

DAS: conceituação, curadoria de dados, investigação, visualização, escrita -rascunho original

Declaração de conflito: nada foi declarado. 